\title{
Nanoplasma-Enabled Picosecond Switches for Ultra-Fast Electronics
}

The broad applications of ultra-wide-band (UWB) signals and $\mathrm{THz}$ waves in quantum measurements ${ }^{1,2}$, imaging and sensing techniques ${ }^{3,4}$, advanced biological treatments ${ }^{5}$, and very-high datarate communications ${ }^{6}$ have drawn extensive attention to the ultrafast electronics. In such applications, high-speed operation of electronic switches is challenging especially when high-amplitude output signals are required ${ }^{7}$. For instance, although field-effect and bipolar junction devices present good controllability and robust performance, their relatively large output capacitance with respect to their ON-state current significantly limits their switching speed ${ }^{8}$. Here we demonstrate a novel on-chip all-electronic device concept based on nano-scale plasma (nanoplasma) that enables picosecond switching of electric signals with a wide range of power levels. The very high electric field in the small spatial scale of the nanoplasma leads to an ultra-fast electron transfer, resulting in extremely short time responses. Ultra-fast switching speed, higher than $10 \mathrm{~V} / \mathrm{ps}$ was achieved, which is about two orders of magnitude larger than that of field-effect transistors and more than ten times faster than the fastest electronic switch nowadays. Extremely short rise-times were measured down to 5-ps which was limited by the employed measurement set-up. By integrating these devices with dipole antennas, high-power $\mathrm{THz}$ signals with $P f=600 \mathrm{~mW}$. $\mathrm{THz}^{2}$ were emitted, which is significantly larger than state-of-the-art in solidstate electronics. Ease of integration as well as compactness of the nanoplasma switches could open new horizons in several fields, such as imaging, sensing, communications, and biomedical applications.
Nanometer-scale transistors based on III-V compound semiconductors, such as GaAs, InAs, and InP, are at the heart of many high-speed and high-frequency electronic systems ${ }^{10}$. Due to their high electron mobilities, these devices exhibit very high small-signal cut-off frequencies, in the terahertz range ${ }^{11}$. However, the high-frequency large-signal performance of transistors is still a challenge, since it is severely limited by the output capacitance $C_{\text {out }}$, electron saturation velocity and critical electric field ${ }^{12}$. The maximum switching speed of a transistor (Fig. 1a) with saturation current $I_{\max }$ is limited to

$$
\left.\frac{\mathrm{d} v}{\mathrm{~d} t}\right|_{\max }=\frac{I_{\max }}{2 C_{\mathrm{out}}} .
$$

which leads to a power $(P)$-frequency $(f)$ trade-off, called $P f^{2}$ limit (Fig. 1b)

$$
\left.P f^{2}\right|_{\max }=\frac{\left(\mathrm{d} v /\left.\mathrm{d} t\right|_{\max }\right)^{2}}{8 \pi^{2} Z}
$$

where $Z$ is the load resistance. Equation (1) is a self-normalized term, independent from device size and gate length, that results in almost similar values of less than $1 \mathrm{~V} / \mathrm{ps}$, for either power or RF devices (Fig. 1c). For example in lateral devices, normalized saturation currents and output capacitances are in range of $1 \mathrm{~mA} / \mu \mathrm{m}$ and $1 \mathrm{fF} / \mu \mathrm{m}$, respectively, resulting in switching speeds of $0.5 \mathrm{~V} / \mathrm{ps}$. Johnson's figure-of-merit $(J F O M)^{12}$ takes into account the breakdown voltage and smallsignal cut-off frequency of devices, although these parameters are obtained from two different measurements at two separate operating points ${ }^{13}$. As shown in Figure 1d, JFOM indicates switching speeds of even less than $1 \mathrm{~V} / \mathrm{ps}$ for GaAs
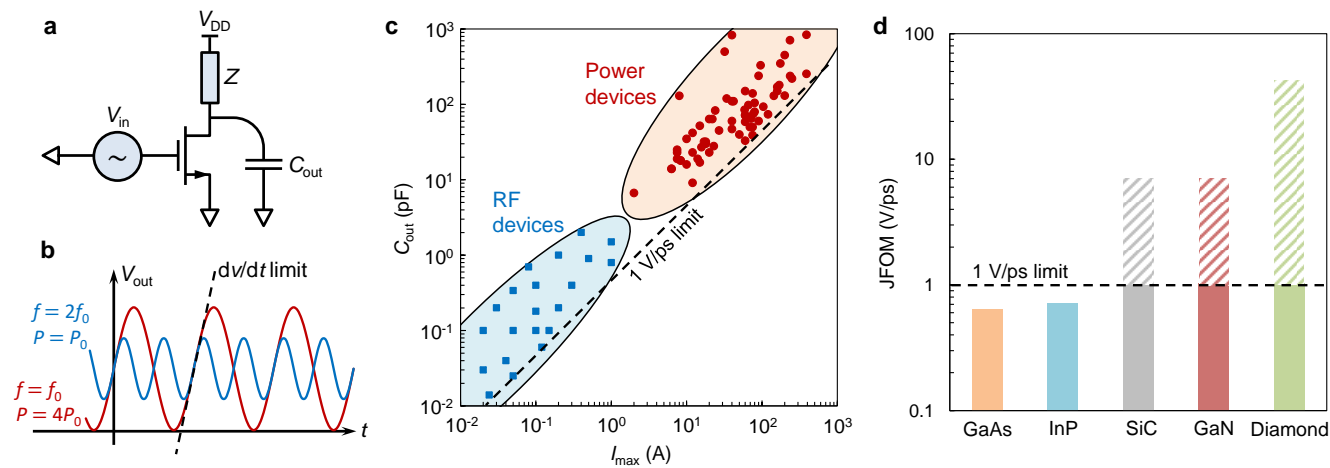

Figure 1 | Switching speed limitation in solid-state electron devices. a, Simple circuit schematic including a transistor with output capacitance $C_{\text {out }}$ and load $Z$. The switching speed of the device is limited to $/ \mathrm{max} / 2 C_{\text {out. }} \mathbf{b}, \mathrm{d} v / \mathrm{d} t$ limitation causes the $P f^{2}$-limit in solid-state electronics. The red curve has double the amplitude and half the frequency of the blue curve, resulting in the same $P f^{2}$, while both are limited to the switching speed. $\mathbf{c}$, Output capacitance versus maximum current for commercial and research devices from the literature and datasheets, both for power and RF applications, showing the $1 \mathrm{~V} / \mathrm{ps}$ limit. In practice, switching speeds as high as $0.5 \mathrm{~V} / \mathrm{ps}$ have been demonstrated. $\mathbf{d}$, JFOM for some of the highperformance semiconductor materials. GaAs and InP are limited to the JFOM, while Cout-limited rise-rate of $1 \mathrm{~V} / \mathrm{ps}$ restricts the performance of SiC, $\mathrm{GaN}$, and Diamond. 
and $\ln \mathrm{P}$, as the semiconductors with the highest electronmotilities and main candidates for ultra-fast electronics (Fig. 1d). As described in Eq. (2), the maximum switching speed of $1 \mathrm{~V} / \mathrm{ps}$ corresponds to an output power of $2.5 \mathrm{~mW}$ at $1 \mathrm{THz}$ for $Z=50 \Omega$. Such a limitation can be seen, not only in the performance of power amplifiers, but also in other solid-statebased approaches including frequency multipliers and negative resistance oscillators (Extended Data Fig. 1). In practice, several phenomena happening at high-frequency and small-scales, such as RF $g_{\mathrm{m}}$ collapse $^{14}$, short-channel effects ${ }^{15}$, and surface traps $^{16}$ result in considerably lower-than-expected powers at the low-THz band (0.1-1 THz).

Plasma devices, such as gas discharge tubes, have nearlyideal ON state providing extremely-high current-densities and do not exhibit the $C_{\text {out }}$-limited switching speed. The dynamic performance of these devices, however, is severely limited by their relatively low electric-field and the considerable electronscattering between two terminals, which result in nanosecondrange switching times ${ }^{17}$. Here we use formation of nanoplasma (Fig. 2a) in atmospheric air to realize an ultra-fast switch that overcomes the switching-speed limitation of solid-state electron devices as well the large-scale plasma switches. As shown in Figure $2 b$, the switch is normally OFF, until a voltage higher than the threshold $V_{\mathrm{TH}}$ is applied. The nano-gap allows an extremely rapid electron transport in the very-high-field region, which results in an ultra-fast switching from an isolated to conducting state, with extremely high ON/OFF ratio. Figure 2c presents the modified Paschen curve ${ }^{18}$ showing the threshold voltage versus gap distance. For small-scale devices, where the origin of plasma formation is either by electric-field emission ( $5 \mathrm{~nm}<g<5 \mu \mathrm{m}$ ) or tunneling $(g<5 \mathrm{~nm}$ ), much higher electric fields than in large-scale plasma devices can be achieved ${ }^{19}$. This results in extremely fast time responses, much faster than that in large-scale plasma devices (with normally nanosecond-range switching times) ${ }^{17}$ (Figure $2 \mathrm{~d}$ ).

The structure of nanoplasma switches is very simple, consisting of metal pads separated by a nanometer-scale gap (Figs. 3a,b), which is compatible with planar fabrication processes and even
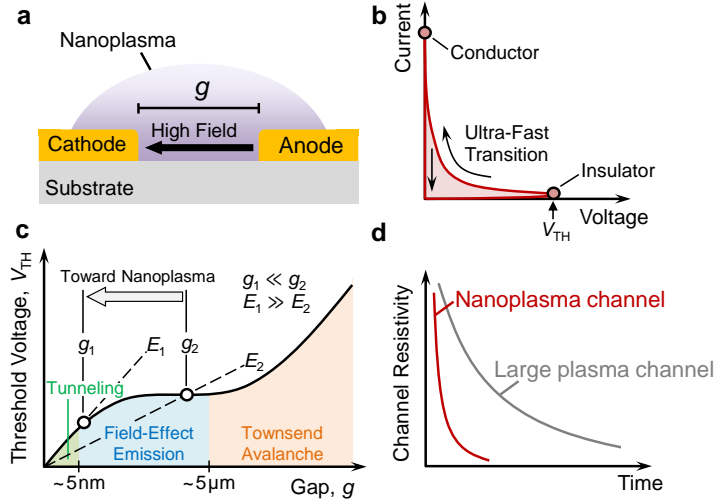

Figure 2 | Concept of nanoplasma switch. a, Schematic of the structure of a nanoplasma switch with gap length $g$. The switch is normally OFF, and becomes $\mathrm{ON}$ at threshold voltage $V=V_{\mathrm{TH}}$ by electron transport in the high-electric-field region. $\mathbf{b}$, current-voltage characteristics of a nanoplasma switch. The switch has two stable phases: Insulating and conducting. The transition from insulating to conducting phases is ultrafast, while the conducting-to-insulating transition is controlled by the external circuit. c, Threshold voltage $V_{T H}$ versus gap distance, corresponding to the modified Paschen law, showing a much larger electric-field for devices with nano-scale gaps. Townsend avalanche is the dominant plasma formation mechanism for $g>5 \mu \mathrm{m}$, while field-effect emission and tunneling are dominant for $5 \mathrm{~nm}<g<5 \mu \mathrm{m}$, and $g<5 \mathrm{~nm}$, respectively. $\mathbf{d}$, The higher electric field in a shorter gap distance results in a much faster electron transport for nanoplasma devices.
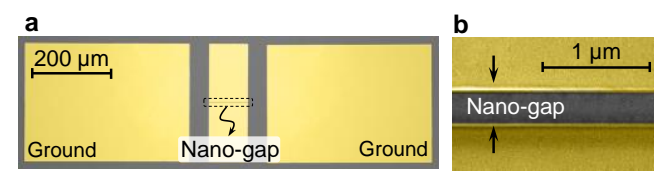

d
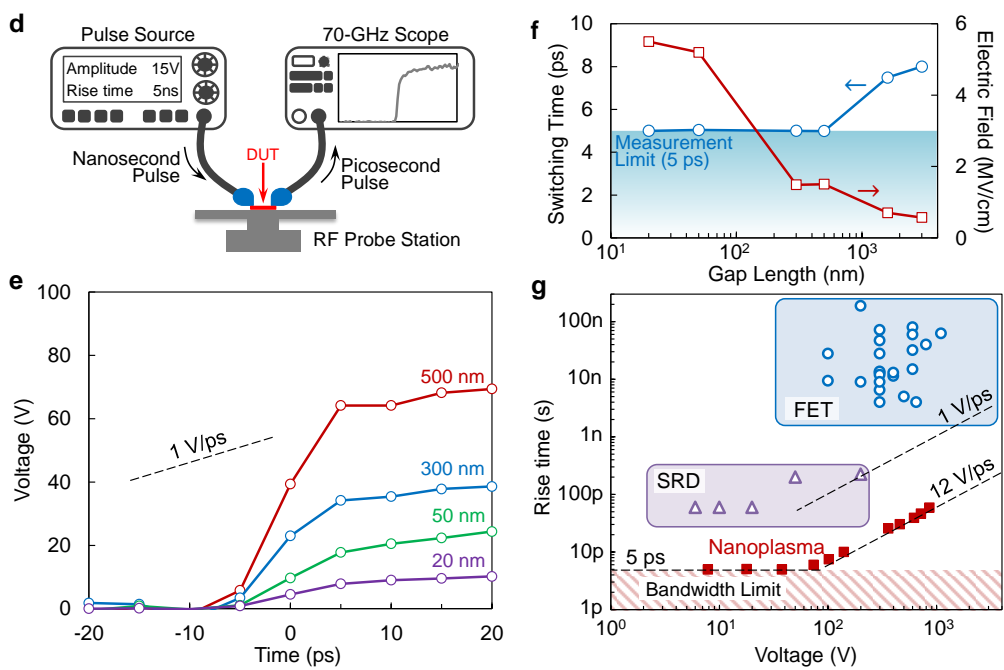

Figure 3 | Implementation of nanoplasma switches. a, Micrograph of a nanoplasma switch integrated with RF pads, and $\mathbf{b}$, a scanning electron microscope image (SEM) of the nano-gap. c, Fabricated devices on Kapton showing their possible integration to flexible substrates. d, Schematic of the experimental set-up for switching characterization of the nanoplasma devices. Time-domain measurements were carried-out with a DPO70000SX 70-GHz Tektronix oscilloscope, which together with the $67 \mathrm{GHz}$ cables and RF probes, limited the rise-time measurements to 6 ps. e, Measured switching waveforms showing 6-ps rise time of high voltage signals. The measured rise time is limited by the set-up bandwidth. f, Measured (blue) together with the electric field in the nano-gap (red). After de-embeding the effect of cable and RF probe, a 5-ps rise-time was obtained (Extended Data Fig. 2). Switching-time measurements in time domain are limited by the oscilloscope bandwidth. $\mathbf{g}$, Benchmark of the risetime/switching-voltage of nanoplasma versus other state-of-the-art electronic devices. 


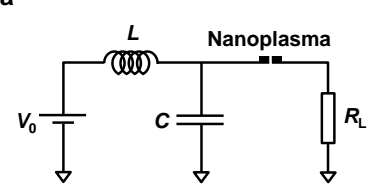

C

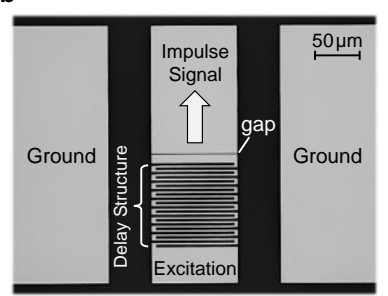

d
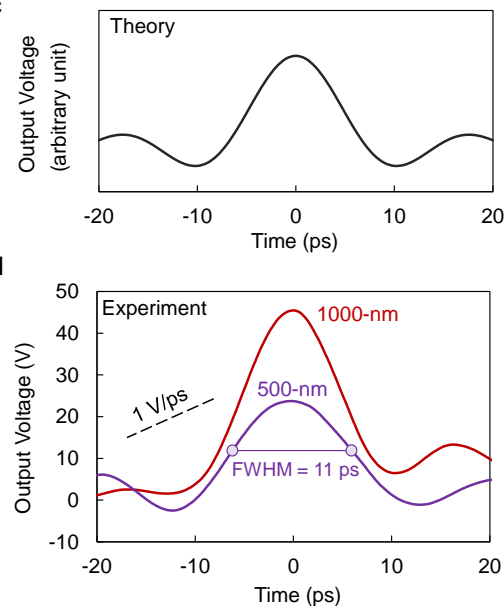

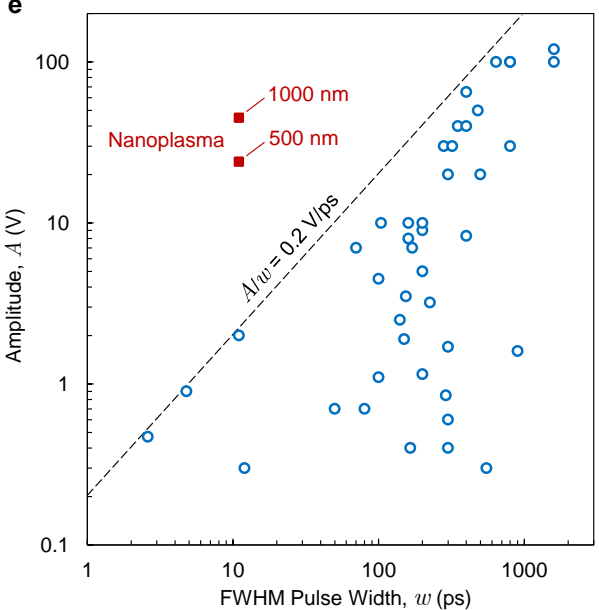

Figure 4 | Impulse generation using nanoplasma switch. a, Simple circuit topology enables nanoplasma to generate ultra-fast impulse signals. The capacitor $C$ is charged through the voltage source $V_{0}$ in series with inductor $L$, up to the $V_{\text {TH }}$ of the nanoplasma device. At this point, the energy stored in $C$ is discharged into the load $R$. The time-constant $\sqrt{L C}$ is higher than the output time-constant $R_{L} C$ so that at a switching event, the capacitor $C$ is discharged alone to the load, without the previous stage being involved (which includes RF probes and cables), resulting in the generation of an impulse. $\mathbf{b}$, Optical image of a monolithically integrated circuit fabricated to provide a charging delay time between the gap and the excitation. c, An ideal impulse signal with arbitrary amplitude after filtering with an ideal 70-GHz low-pass filter. d, Measurement results after de-embedding for devices shown in part b, with two gap sizes 500-nm and 1000-nm, showing a very high amplitude and waveforms close to the ideal impulse (sinc function). e, Benchmark of amplitude versus full-width-at-half-maximum (FWHM) of the generated pulses against with reported data in the literature and datasheets. For the references of the data points, please refer the supplementary information.

possible integration to flexible substrates (Fig. 3c). A very high bandwidth experimental set-up, shown in Fig. 3d, was used to characterize the switching dynamics of fabricated nanoplasma devices. A nanosecond pulse generator supplies the input pulse, and once the input signal reaches the $V_{T H}$, the nanoplasma switch turns ON, passing the signal to the $50-\Omega$ termination of oscilloscope. Figure $3 e$ shows the measured switching waveforms. Comparing the very sharp switching transients of nanoplasma devices with state-of-the-art switching speed in solid-state electronics, reveals the outstanding performance of these devices for ultra-fast electronics, especially for applications requiring large signals. The measured rise-time (Fig. 3f) for small scale devices, with gap lengths down to $20 \mathrm{~nm}$, reached the measurement bandwidth limit of 6-ps, which after de-embedding the effect of cables and attenuators, resulted in a rise-time of 5 ps (Extended Data Fig. 2). Figure $3 g$ shows measured rise-time versus amplitude for the fabricated devices in comparison to reported data for the fastest electronic switches including field-effect transistors (FETs) and step-recovery diodes (SRDs). While the switching speed of solid-state electronics is below 1 $\mathrm{V} / \mathrm{ps}$ (in agreement with the theoretical prediction in Fig. 1), nanoplasma devices reached much higher values while they are not limited any more by the parasitic capacitance (Extended Data Fig. 3). In such a comparison we should note that FETs, as three-terminal devices, are capable of generating rise and fall edges with the same speed, while in other highperformance electron devices, including step-recovery-diode $(\mathrm{SRD})^{20}$ or silicon-avalanche-diode $(\mathrm{SAS})^{21}$, as two-terminal devices, a single fast switching-edge is provided. Nanoplasma switch provides a significantly faster switching time at higher voltage levels which enables generating much faster UWB signals at higher power levels. A good consistency in the switching transient as well as a durable performance under harsh switching conditions are other beneficial aspects of nanoplasma devices for high-power ultra-fast electronics (Extended Data Figs. 4 and 5).

Generation of impulse and square-shape signals is enabled by devices providing a single fast switching transient based on techniques such as discharging the energy stored in a capacitor (Fig. 4a), inductor, transmission line, etc., or by utilizing delaylines ${ }^{22,23}$. The switching time and voltage/current capability of the switch limits the minimum rise (fall)-time and pulse-width, as well as the maximum peak power of generated signals. Figure $4 \mathrm{~b}$ shows a simple monolithically integrated circuit fabricated to demonstrate the impulse generation using nanoplasma devices. The delay structure between the excitation port and the nano-gap does not let the input transmission line (including RF probe and cable) to discharge through the gap immediately after switching. The experimental set-up shown in Fig. 3d was used to characterize the monolithically integrated circuit. Figure 4c shows an ideal impulse with arbitrary amplitude after passing an ideal $70-\mathrm{GHz}$ low-pass filter (corresponding to the bandwidth of the used oscilloscope), which in fact, is a sinc function. The obtained waveforms for devices with two different gap sizes are shown in Fig. 4d. Nanoplasma devices presented a much higher amplitude and shorter pulses than any other electron device in 

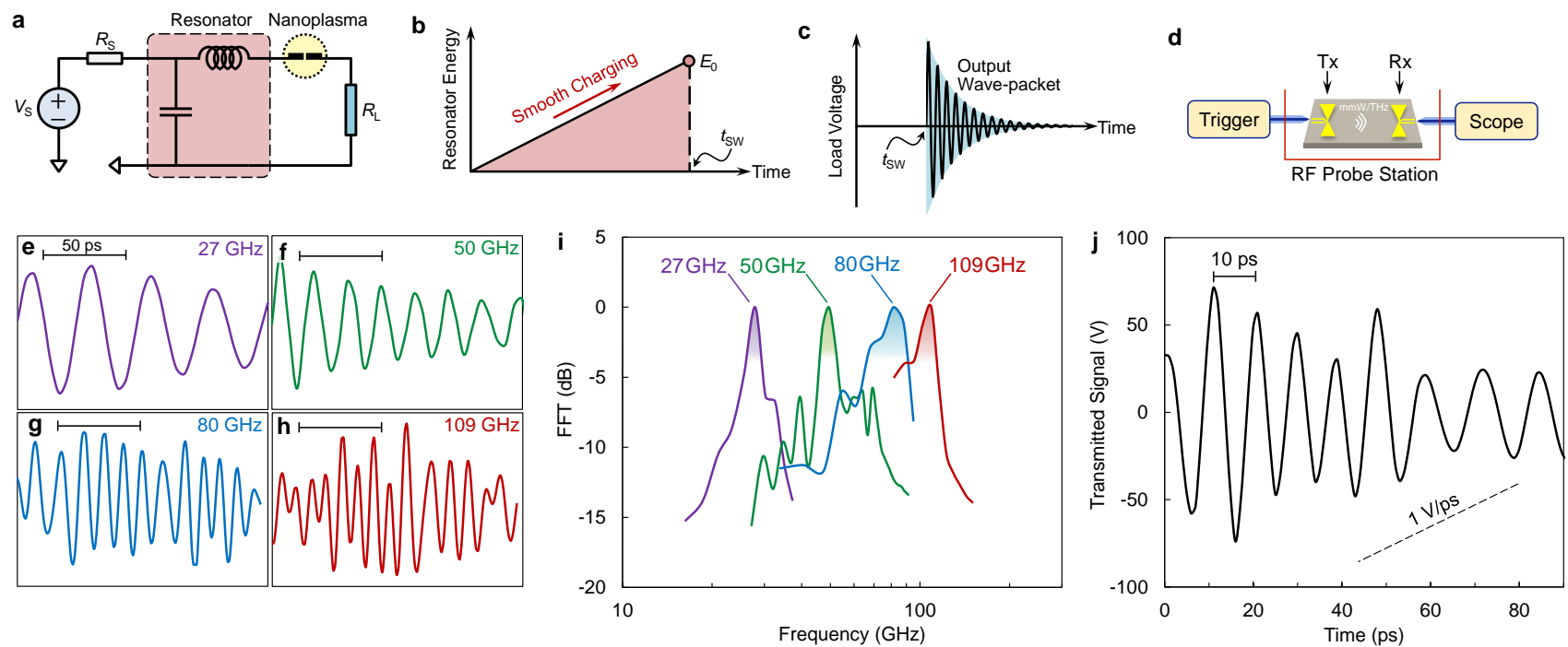

Figure 5 | Nanoplasma-based mm-wave/THz source. a, Implemented circuit diagram of mm-wave/THz source realized by nanoplasma switch. b. The voltage source $V_{S}$ smoothly charges a fast resonator, which is excited at $V=V_{T H}$ when the nanoplasma switch turns ON with picosecond transition time. The resonance frequency of the resonator determines the central frequency $\left(f_{0}\right)$ of the generated pulse and the switching transition of the nanoplasma switch should be small enough $\left(\lesssim 1 / f_{0}\right)$ to excite the resonator. The energy is transferred to the load in a short time after switching time tsw. c, Illustration of the generated mm-wave/THz pulse at $t=t$ sw. d, Schematic of the experimental set-up. The THz pulse is emitted by a transmitter antenna connected to the nanoplasma switch and the received wave is measured by an ultra-high-frequency oscilloscope. Measured waveforms with bowtie antennas with different sizes (the transmitter and receiver antennas have the same size) resulting in central frequencies of e, $27 \mathrm{GHz}, \mathbf{f}, 50 \mathrm{GHz}, \mathbf{g}, 80 \mathrm{GHz}$, and $\mathbf{h}, 109 \mathrm{GHz}$. i, Frequency spectrum of the received signals for four different antenna sizes showing the flexibility of the method in generating high-frequency signals at different frequencies, (up to the measurement limit of our set-up). j, Calculated radiated signal from the transmitter antenna, based on the measured $S_{21}$ after de-embedding the effect of cables and RF probe, showing a high average peak power of $50-\mathrm{W}$ at $109-\mathrm{GHz}$.

the literature (Fig. 4e) which shows their outstanding potential for UWB systems.

The ultra-fast performance of the proposed devices also enables the generation of $\mathrm{mm}$-wave and $\mathrm{THz}$ signals at high peak-power levels. As shown in Fig. 5a, a resonator illustrated by an LC tank is smoothly charged (Fig. 5b). When the output voltage reaches the threshold voltage of the nanoplasma switch, it turns ON, discharging the energy stored in the resonator, as a high-frequency wave-packet, into the load (Fig. 5c). The performance of such a $\mathrm{mm}$-wave/THz pulse source is determined by the employed switch as well as the resonator design. Picosecond switching of the nanoplasma enables exciting very high resonance frequencies in the $\mathrm{THz}$ range, and the high-amplitude operation of the switch results in large energy stored in the resonator, leading to high output powers. The resonator design determines the resonance frequency as well as the $\mathrm{mm}$-wave/THz pulse energy. Fig. $5 \mathrm{~d}$ shows the schematic of the high band-width experimental set-up used tocharacterize the wave emission from nanoplasma switches integrated with bowtie antennas, serving as both the resonator and radiating element. An antenna with the same geometry connected to a high frequency oscilloscope was used to receive the radiated wave-packet. Four received waveforms with different antenna sizes resulting in central frequencies of 27 , 50, 80, and $109 \mathrm{GHz}$, are shown in Figs. 5e, f, g, and h, respectively (The maximum chosen frequency was limited by the bandwidth of our $113 \mathrm{GHz}$ oscilloscope). The time scale bars are 50-ps and for the 27-GHz waveform, the second harmonic of the antenna was filtered out. Fig. $5 \mathrm{i}$ shows the FFT of received signals. Based on frequency-domain characterization of the antennas (Extended Data Fig. 6), and after de-embedding the effect of cables and RF probe an average peak power of $50-\mathrm{W}$ at $109-\mathrm{GHz}$ corresponding to $P f^{2}$ of $600 \mathrm{~mW} . \mathrm{THz}^{2}$ was obtained (Fig. 5j). This is more than 200 times larger than the $P f^{2}=2.5 \mathrm{~mW} \cdot \mathrm{THz}^{2}$ limited by $1 \mathrm{~V} / \mathrm{ps}$ switching speed in solid-state electronics. In addition, the very high frequency operation of the proposed devices can have also a significant impact in flexible electronics. While 2D semiconductors are showing promising RF performances, their relatively small mobility severely limits the bandwidth of existing flexible electronic systems ${ }^{24}$. Nanoplasma devices increase the bandwidth of flexible integrated electronic systems, from below $10 \mathrm{GHz}$ in $2 \mathrm{D}$ semiconductors to the $\mathrm{THz}$ band ${ }^{25}$.

Figure 6 demonstrates pulse sharpening with very high repetition rate using nanoplasma devices. The experimental set-up for high repetition-rate pulse sharpening characterization is shown in Fig. 6a. A 10-MHz GaN-based pulse source drives the DUT which is connected to a 1-GHz scope through a stack of attenuators. Figure $6 \mathrm{~b}$ shows how the 

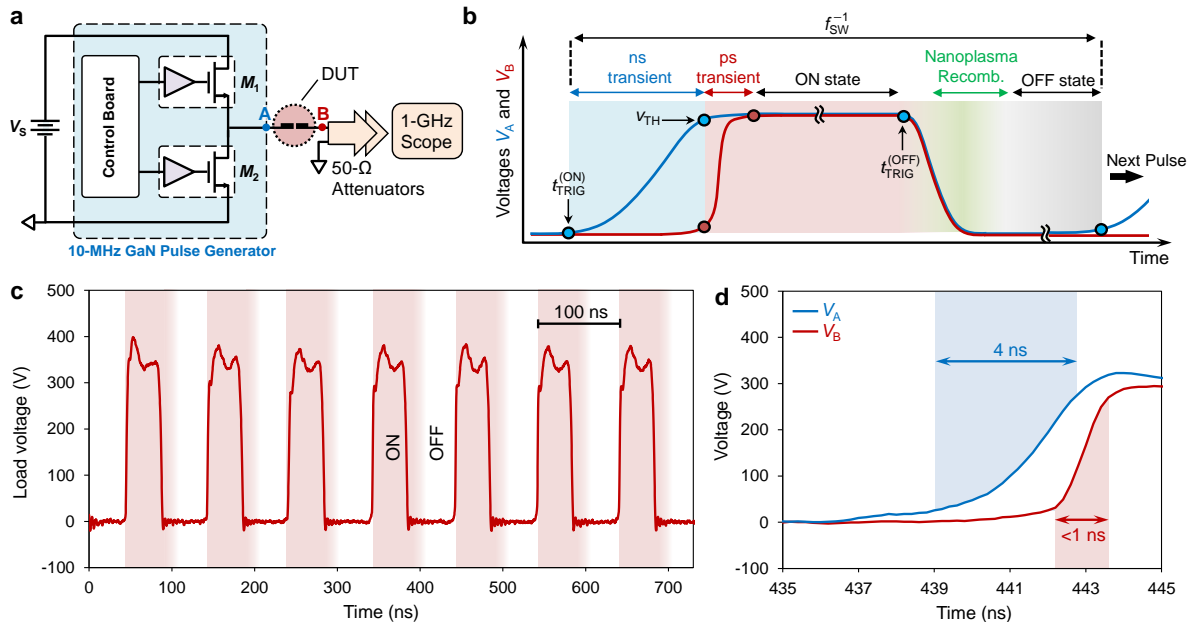

Figure 6 | High-repetition-rate pulse sharpening with nanoplasma switches. a, Schematic of the experimental set-up for repetitive pulse sharpening with nanoplasma devices. A GaN-based half-bridge applies pulses with $10-\mathrm{MHz}$ switching frequency and $50 \%$ duty cycle to the on-chip plasma (DUT) switch, showing the high repetition rate of the nanoplasma switches. The signal is then terminated by $50-\Omega$ port of an oscilloscope after a chain of attenuators with $40-\mathrm{dB}$ attenuation. $\mathbf{b}$, Illustration of waveforms before $\left(V_{\mathrm{A}}\right)$ and after the DUT $\left(V_{\mathrm{B}}\right)$. At $\mathrm{t}=t_{\mathrm{TRIG}}(\mathrm{ON})$ the $\mathrm{GaN}$ pulser generates a pulse (nanosecond rise-time). The DUT is OFF until the voltage reaches $V_{\mathrm{TH}}$, then it turns ON in a very short time (picosecond risetime). The DUT maintains its OFF state until $t_{\mathrm{TRIG}}{ }^{(\mathrm{OFF})}$ when the GaN pulser starts to switches OFF the output voltage. After a recombination time, the DUT retains its OFF state. c, Voltage waveform $\left(V_{B}\right)$ over the $50-\Omega$ load demonstrating $10-\mathrm{MHz}$ high-power switching with DUT. d, Voltage waveforms $V_{A}$ and $V_{B}$, showing the pulse sharpened $V_{B}$ by the nanoplasma (limited to the measurement bandwidth).

output signal of the nanoplasma device follows the input signal. The device turns ON just after the input signal reaches $V_{\mathrm{TH}}$, resulting in a pulse sharpening, which converts the input nanosecond rise-time to a picosecond transition at the output. The switch remains in the ON state until the driver circuit

supplies the signal. When the input signal becomes zero, the ultra-fast recombination of ionized molecules in the nanoplasma recovers the OFF state of the switch. The switching frequency of this experiment is limited by the solidstate pulse generator, however we demonstrated a $20-\mathrm{MHz}$ switching frequency at $390-\mathrm{V}$, together with ultra-fast recombination time of $<20-n s$ which enables to further increase the switching frequency up to $50-\mathrm{MHz}$ (Extended Data Fig. 7). The ultra-high switching speed and the capability of operating at very high repetition rates, unveil the significant potential of nanoplasma switches for ultra-fast electronic systems, especially those operate at high-power levels.

In conclusion, we proposed an integrable device concept that enables picosecond switching of high-amplitude signals. In addition to its simplicity and low fabrication cost, its performance surpasses that of today's ultra-high-speed electron devices. With a proper circuit design, nanoplasma devices enable the generation of ultra-fast high-power step and impulse signals, which are key elements for UWB systems. The use of nanoplasma devices in high-power $\mathrm{THz}$ pulse generation was demonstrated, where high-amplitude peak-topeak voltage exceeding $100-\mathrm{V}$ was emitted at $109-\mathrm{GHz}$, by simply integrating the device with a bow-tie antenna. The tunability of $V_{\mathrm{TH}}$ together with a high-current capability and low capacitance enable nanoplasma devices to operate as highperformance ultra-fast low-parasitic protection unit at the front-end of RF systems, as well as protection for avalanchefree electron devices (Extended Data Fig. 8). The highperformance and simplicity of the proposed devices offer new horizons for the future chip-scale ultra-fast electronics and $\mathrm{THz}$ sources with applications in communications, imaging, sensing and biomedicine, among others.

\section{METHODS}

General. All the measurements where done under a controlled ambient, with temperature and humidity of $22{ }^{\circ} \mathrm{C}$ and $50 \%$, respectively. The effect of temperature and humidity has been considered in physics of large-scale plasma devices, which show low sensitivity to relatively small variations of these parameters $^{26,27}$.

Power-frequency trade-off in transistors since any change in the voltage over the transistor results in charging/discharging of its output capacitance, the maximum $\mathrm{d} v / \mathrm{d} t$ is limited to (1). Considering a sine-wave over drain-source (Fig. 1a)

$$
v=\frac{1}{2} A+\frac{1}{2} A \sin (2 \pi f t)
$$

results in

$$
\left.\frac{\mathrm{d} v}{\mathrm{~d} t}\right|_{\max }=\pi f A
$$

which can be re-written as

$$
\left.\frac{\mathrm{d} v}{\mathrm{~d} t}\right|_{\max }=\pi \sqrt{2 Z} \sqrt{P f^{2}} .
$$

Comparing (1) and (5) results in (2). 
Device fabrication. Nanoplasma devices were fabricated on low-cost 2-inch sapphire substrate. After the initial step of substrate cleaning, 100-nm-thick gold/tungsten on top of 20-nmthick titanium layer was deposited by electron-beam evaporation. Then, an electron-beam lithography step with 50\% ZEP (baked at $180^{\circ} \mathrm{C}$ ) resist followed by ion beam etching was employed to form the nanogaps. A photolithography using 1.4- $\mu$ m-thick $A Z$ nLof 2020 photoresist followed by ion-beam etching was used to form the RF pads. For the e-beam lithography step of devices with higher thickness (500-nm), a 100\% ZEP resist with 1000rpm coating speed corresponds to $650-\mathrm{nm}$ thickness was used. The sample was baked at $180^{\circ} \mathrm{C}$ before and after the coating. For e-beam lithography, we have used the dose from $240 \mu \mathrm{C} / \mathrm{cm}^{2}$ and then 2 min into developer Amyl-Acetate and 1 mins 90:10 MiBK:IPA rinse solution. For devices on flexible substrate (Kapton), we first pasted a Kapton tape on a sapphire substrate. The rest of process flow is similar to the devices on sapphire substrate. The Kapton was removed from the sapphire wafer after the fabrication process.

Parasitic capacitance measurement of nanoplasma devices. As shown in Extended Data Fig. 3, the capacitance of nanoplasma switches was measured to determine their $C_{\text {out }^{-}}$ limited $\mathrm{d} v / \mathrm{d} t$. The capacitance was extracted from measured Sparameters using a Keysight 50-GHz PNA network analyzer N5225A. In a two-port measurement, the scattering parameters were measured. Then, the ABCD parameters were extracted from scattering parameters. The parameter $B$ in $A B C D$ matrix equals to the series impedance $Z(\omega)=1 /(j C \omega)$, from which the capacitance $C$ was extracted.

Antenna characterization. In order to evaluate the performance of nanoplasma-based $\mathrm{mm}$-wave/ $\mathrm{THz}$ radiators and to determine the value of radiated power, we characterized the fabricated antennas, using Anritsu 110-GHz VNA. Using a two-port measurement, the reflection and transmission scattering parameters between transmitter and receiver antennas were measured (Extended Data Fig. 6). The transmitted power was determined based on the measured transmission between two antennas, as well as the insertion loss in the $110-\mathrm{GHz}$ coaxial cables and the 110-GHz RF probe.

De-embedding procedure. To eliminate the effect of measurement set-up chain (RF probe, cables, etc.) from the measurements we used the s-parameters of different parts. In particular for the switching transient measurements, the measured s-parameters of RF probe and cable given by the manufacturers were used (Extended Data Fig. 2). To compute the overall s-parameters matrix, we transferred the premeasured s-parameter matrices to $A B C D$ matrices, where the total $A B C D$ matrix can be simply calculated as the product of two $A B C D$ matrices. Then, the obtained $A B C D$ matrix was converted to s-parameters matrix, and the $S_{21}$ was used as the transfer function of the measurement chain. Then, by calculating $\mathrm{F}^{-1}\left[S_{21}(\omega) \cdot \mathrm{F}[v(t)]\right]$, where $v(t)$ is the measured signal with oscilloscope and $F$ is the fast Fourier transform (FFT), we extracted the de-embedded signal. A similar procedure was used for obtaining the radiated signal presented in Fig. 4j. In this case, there are more elements in the measurement chain: transmission antenna, channel between two antennas, receiving antenna, RF probe, and cables. The measured s-parameters of the antenna (Extended Data Fig. 6) covers the first three elements.
Considering a chain including the measured and reported sparameters, the total s-parameter matrix was extracted, where $S_{21}(\omega)$ is the transfer function. By using this transfer function we extracted the radiated signal.

10-MHz GaN-based pulse generator. The experimental system consists of power stage and a control stage. The power stage is a $10 \mathrm{MHz}$ half-bridge circuit that uses two $600 \mathrm{~V} / 70 \mathrm{~m} \Omega$ LMG3410R70 GaN power transistors with integrated drivers from Texas Instruments. The PCB consists of 4 layers whose layout is highly optimized to minimize the parasitics at $10 \mathrm{MHz}$ switching. The dc-link voltage of $400 \mathrm{~V}$ for the power-stage is provided by a Magna-Power TSD800-18/380 high-voltage DC power supply. The control stage, which is galvanically isolated from the power stage, provides the gating signals for the transistors at $10 \mathrm{MHz}$ using a Texas Instruments' F28379d highperformance Digital Signal Processor (DSP).

Lifetime evaluation. High current density levels in the proposed devices could be a possible limitation to the lifetime of the switches due to electromigration ${ }^{28}$. The potential effect is the progressive deformation of the gap as the device operates, making the gap wider and leading to a change in $V_{\mathrm{TH}}$. As a result, the relative $V_{T H}$ shift can be considered to quantify the device degradation. To evaluate its lifetime under operation, the switch was submitted to repetitive short circuits, resulting the highest possible current density (as the main drive for electromigration), and the degradation after the $n^{\text {th }}$ short circuit was defined as:

$$
\text { degradation after } n \text {th short circuit }=\frac{V_{\mathrm{TH}}[n]-V_{\mathrm{TH}}[0]}{V_{\mathrm{TH}}[0]}
$$

where $V_{\mathrm{TH}}[n]$ is the threshold voltage after the $n^{\text {th }}$ short. Because of the repetitive high current levels and the short-circuit nature of the experiment (instead of $50-\Omega$ loads), the measurement could not be performed using RF probes (the probes damaged quicker than the devices under test). Thus devices with total size of $4 \mathrm{~mm}$ $x 4 \mathrm{~mm}$ where fabricated to be directly connected to a circuit (Extended Data Fig. 7a with $L=1 \mathrm{mH}$ ). Devices with 700-nm-gap were submitted to repetitive 15-ns-long short circuits with dissipated energy of $3 \mu \mathrm{J}$ per pulse. The dissipated power inside the switch at each short circuit pulse is shown in the Extended Data Fig. 5a. The test was performed for devices with two different pad metal thicknesses (100-nm and 500-nm), both with sputtered tungsten (Extended Data Fig. 5b).

\section{References}

1. Benea-Chelmus, I.-C., Settembrini, F. F., Scalari, G. \& Faist, J. Electric feld correlation measurements on the electromagnetic vacuum state. Nature 568, 202-206 (2019).

2. Kroll, J. et al. Phase-resolved measurements of stimulated emission in a laser. Nature 449, 698-701 (2007).

3. Cocker, T. L., Peller, D., Yu, P., Repp, J. \& Huber, R. Tracking the ultrafast motion of a single molecule by femtosecond orbital imaging. Nature 539, 263-267 (2016).

4. Jelic, V. et al. Ultrafast terahertz control of extreme tunnel currents through single atoms on a silicon surface. Nat. Phys. 13, 591-598 (2017).

5. Rossi, A., et al. Mechanisms and immunogenicity of nsPEFinduced cell death in B16F10 melanoma tumors. Sci Rep. 9, 431 (2019). 
6. Koenig, S. et al. Wireless sub-THz communication system with high data rate. Nat. Photon 7, 977-981 (2013).

7. Sengupta, K., Nagatsuma, T. \& Mittleman, D. Terahertz integrated electronic and hybrid electronic-photonic systems. Nat. Electron. 1, 622-635 (2018).

8. Baliga, B. J. Fundamentals of power semiconductor devices. Springer Science \& Business Media, (2010).

9. Auston, D. H. Picosecond optoelectronic switching and gating in silicon. Appl. Phys. Lett. 26, 101-103 (1975).

10. del Alamo, J. A. Nanometre-scale electronics with III-V compound semiconductors. Nature 479, 317-323 (2011).

11. Mei, X. et al. First demonstration of amplification at $1 \mathrm{THz}$ using 25-nm InP high electron mobility transistor process. IEEE Electron Device Lett. 36, 327-329 (2015).

12. Johnson, E. O. Physical limitation on frequency and power parameters of transistors. IEEE Int. Conv. Record, 27-34 (1965).

13. Zheng, X., Guidry, M., Li, H., Romanczyk, B., Ahmadi, E., Hestroffer, K., Wienecke, S., Keller, S., \& Mishra, U. K. N-polar GaN MISHEMTs on sapphire with a proposed figure of merit $f_{\max } \cdot V_{\mathrm{DS}, \mathrm{Q}}$ of $9.5 \mathrm{THz} . \mathrm{V}$. 75th Annu. Device Res. Conf., 1-2 (2017).

14. Lee, D. S. et al. $245 \mathrm{GHz} \operatorname{InAIN/GaN~HEMTs~with~oxygen~}$ plasma treatment. IEEE Electron Device Lett. 32, 755-757 (2011).

15. Young, K. K. Short-channel effect in fully depleted SOI MOSFET's. IEEE Trans. Electron Devices 36, 399-402 (1989).

16. Vetury, R., Zhang, N. Q., Keller, S. \& Mishra, U. K. The impact of surface states on the DC and RF characteristics of AIGaN/GaN HFET's. IEEE Trans. Electron Devices 48, 560566 (2001).

17. G. A. Mesyats, Pulsed Power. Norwell, MA: Kluwer, 2005.

18. Loveless, A. M., Garner, A. L., Scaling laws for gas breakdown for nanoscale to microscale gaps at atmospheric pressure. Appl. Phys. Lett. 108, 234103 (2016).

19. Wallash, A., Levit, L. Electrostatic discharge/electrical overstress susceptibility in MEMS: A new failure mode. Proc. SPIE 4180, 30-39 (2000).

20. Moll, J.L., \& Hamilton, S.A. Physical modeling of the step recovery diode for pulse and harmonic generation circuits. Proc. IEEE 57, 1250-1259 (1969).

21. Prager, H. J., Chang, K. K. N., \& Weisbrod, S. High-power, high-efficiency silicon avalanche diodes at ultra-high frequencies. Proc. of the IEEE 55, 586-587 (1967).

22. Orlenko O.A. UWB pulse generators. Int. Conf. on Ultrawideband and Ultrashort Impulse Signals, 75-77 (2012).

23. Nguyen C. \& Han. J. Design of delay-line SRD impulse generator. In Time-Domain Ultra-Wideband Radar, Sensor and Components: Theory, Analysis and Design, Springer Science \& Business Media, 27-33 (2014).
24. Zhang, X. et al. Two-dimensional $\mathrm{MoS}_{2}$-enabled flexible rectenna for Wi-Fi-band wireless energy harvesting. Nature 566, 368-372 (2019).

25. Gao, Q., Zhang, Z., Xu, X., Song, J., Li, X., Wu, Y., Scalable high performance radio frequency electronics based on large domain bilayer $\mathrm{MoS}_{2}$. Nat. Commun. 9, 4778 (2018).

26. Uhm, H. S., Jung, S. J. \& Kim, H. S. Influence of gas temperature on electrical breakdown in cylindrical electrodes. J. Korean Phys. Soc. 42, 989-993 (2003).

27. Allen, K. R., \& K. Phillips. Effect of humidity on the spark breakdown voltage. Nature 183, 174-175 (1959).

28. Ho, P. S. \& Kwok, T. Electromigration in metals. Rep. Prog. Phys. 52, 301-348 (1989).

29. Radisic, V., Leong, K. M. K. H., Mei, X., Sarkozy, S., Yoshida, W., \& Deal, W. R. Power amplification at $0.65 \mathrm{THz}$ using $\operatorname{InP}$ HEMTs. IEEE Trans. Microw. Theory Tech. 60, 724-729 (2012).

30. Leong, K. M. K. H. et al. A $0.85 \mathrm{THz}$ low noise amplifier using InP HEMT transistors. IEEE Microw. Wireless Compon. Letts. 25, 397-398 (2015).

31. Shingubara, S., Nakasaki, Y., \& Kaneko, H. Electromigration in a single crystalline submicron width aluminum interconnection. Appl. Phys. Lett. 58, 42-44 (1991).

32. Tonouchi, M. Cutting-edge terahertz technology. Nat. Photon. 1, 97-105 (2007).

33. Rieh, J.-S., Yoon, D., \& Yun, J. An overview of solid-state electronic sources and detectors for Terahertz imaging. 12th IEEE Int. Conf. Solid-State Integr. Circuit Technol., 1-4 (2014).

34. Yang, C., Liu, P. \& Huang, X. A novel method of energy selective surface for adaptive HPM/EMP protection. IEEE Antennas Wireless Propag. Lett. 12, 112-115 (2013).

35. Gurevich, V. Protecting Electrical Equipment: Good practices for preventing high altitude electromagnetic pulse impacts. Walter de Gruyter GmbH \& Co KG, 2019.

36. Zhou, C. H. et al. Vertical leakage/breakdown mechanisms in AIGaN/GaN-on-Si devices. IEEE Electron Device Lett. 33 (8), 1132-1134 (2012). 

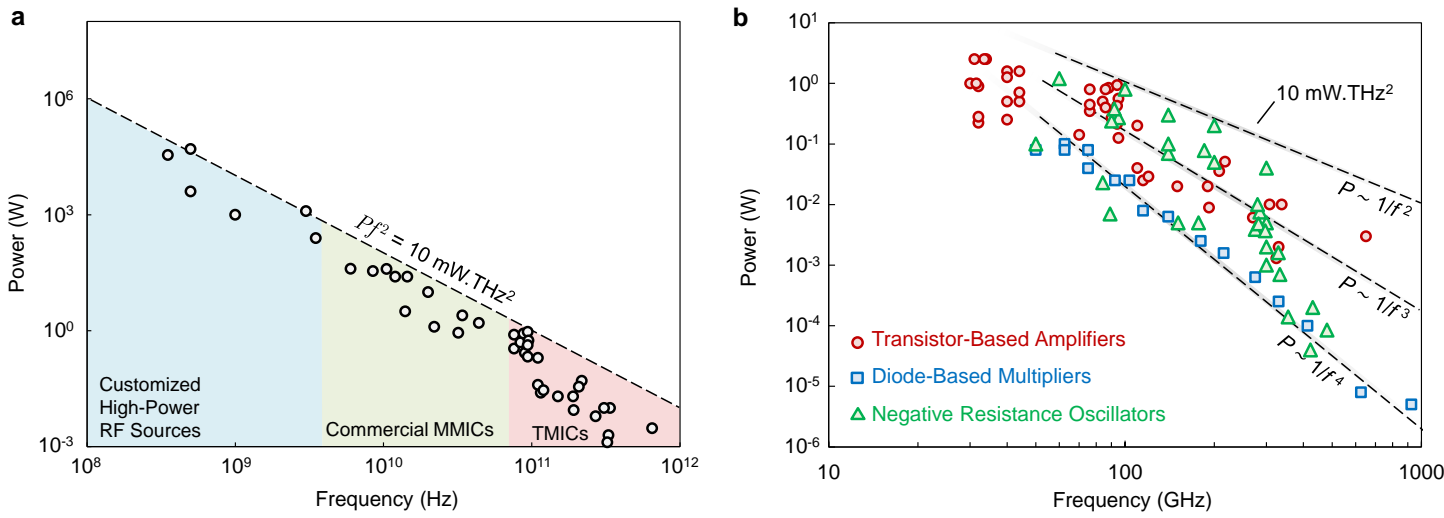

Extended Data Figure 1 | Benchmark of state-of-the-art power amplifiers. a, Power-versus-frequency trade-off in power amplifiers reveals a decay of output power as frequency increases, resulting in a constant $P f$. It should be noted that the limit of $2.5 \mathrm{~mW} . \mathrm{THz}^{2}$ in (2) has been obtained for a single transistor. With power combination techniques in power amplifiers, relatively higher powers (still below $\sim 10 \mathrm{~mW}$. THz ${ }^{2}$ ) can be obtained. At THz band, however, such a power combination becomes very challenging, especially if a high bandwidth is required. As an example of state-of-the-art performance, Radisic et al. ${ }^{29}$ achieved $1.7-\mathrm{mW}$ at $650-\mathrm{GHz}$ in a single power amplifier corresponding to $P f^{2}=0.7 \mathrm{~mW}$. THz ${ }^{2}$. Another work presented by Leong et al. ${ }^{30}$ reported $0.93-\mathrm{mW}$ at $0.85-\mathrm{THz}$ showing $P f^{2}=0.67 \mathrm{~mW} . \mathrm{THz}^{2}$. b. Power-versus-frequency trade-off in different solid-state-based mm-wave/THz sources showing the generality of the decrease in power at high frequencies. For the references of the data points, please refer the supplementary information. 

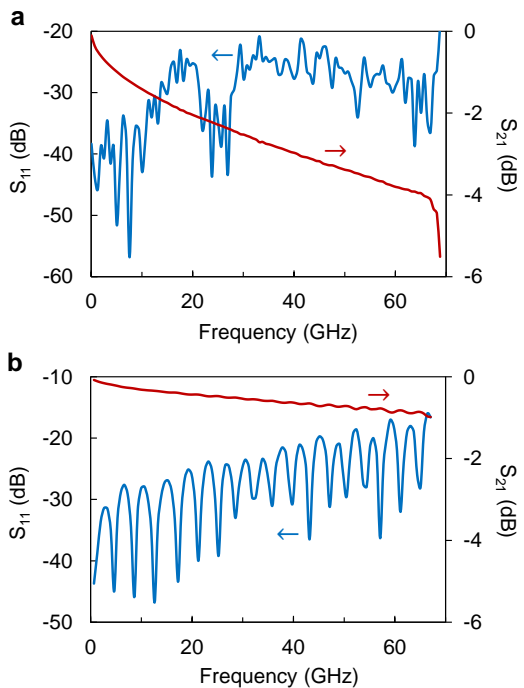
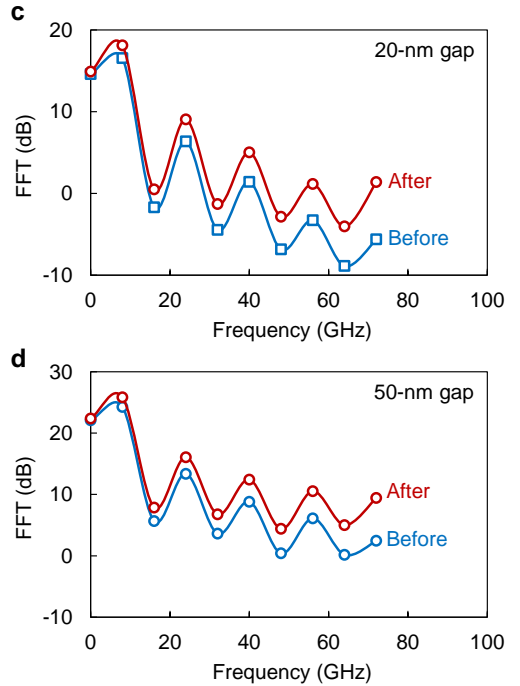

e
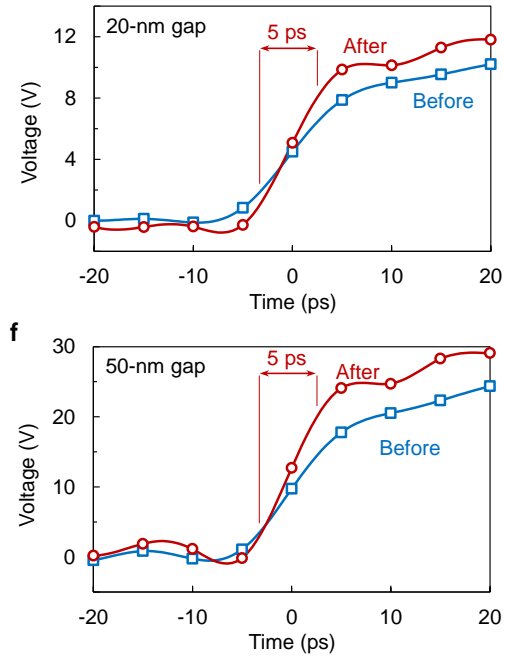

Extended Data Figure 2 | De-embedding cabling and probing effects from time-domain measurements. Measured reflection and transmission scattering parameters for the used a, high-frequency coaxial cable and $\mathbf{b}$, ground-signal-ground (GSG) RF probe, reported by the manufacturer. $\mathbf{c}$, d, FFT of the measured signal (blue) and the obtained FFT after de-embedding (red) for 20-nm and 50-nm-wide gap devices. e, $\mathbf{f}$, The measured (blue) and de-embedded (red) waveforms for 20-nm and 50-nm-wide gap devices showing 5-ps rise-time corresponding to the 70-GHz bandwidth of the oscilloscope. 
a
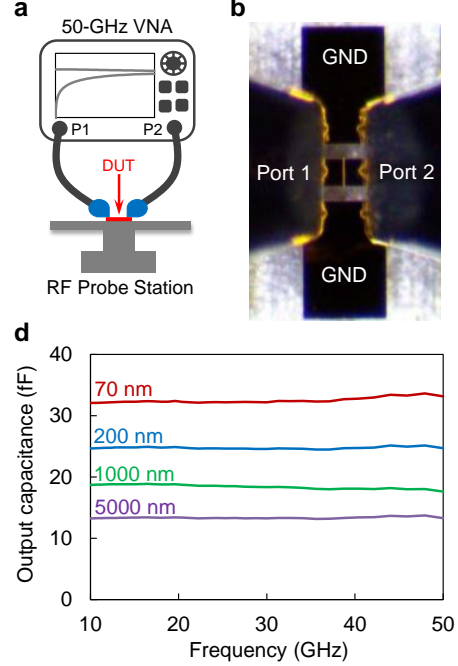

C
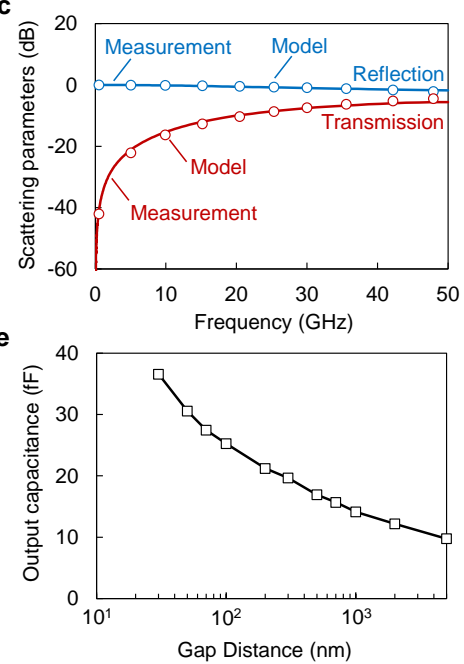

Extended Data Figure 3 | Parasitic capacitance characterization of nanoplasma devices. a, Schematic of the experimental set-up for capacitance measurement. b, Optical image of a device under test in series configuration with two ports of the parametric network analyzer. c, Measured (solid lines) and modeled (discrete points) reflection $\left(S_{11}=S_{22}\right)$ and transmission $\left(S_{21}=S_{12}\right)$ coefficients through a 100-nm-gap nanoplasma device. The device was simply modeled as a 25-fF capacitance. d, Extracted capacitance versus frequency for devices with $\mathrm{g}=70 \mathrm{~nm}, 200 \mathrm{~nm}$, $1000 \mathrm{~nm}$, and $5000 \mathrm{~nm}$. e, Extracted capacitance versus gap distance. The small parasitic capacitances lead to very high dv/dt-limit for nanoplasma devices e.g. $42 \mathrm{~V} / \mathrm{ps}$ for 500 nm-gap devices. The capacitance can be further decreased by shrinking the device width. 

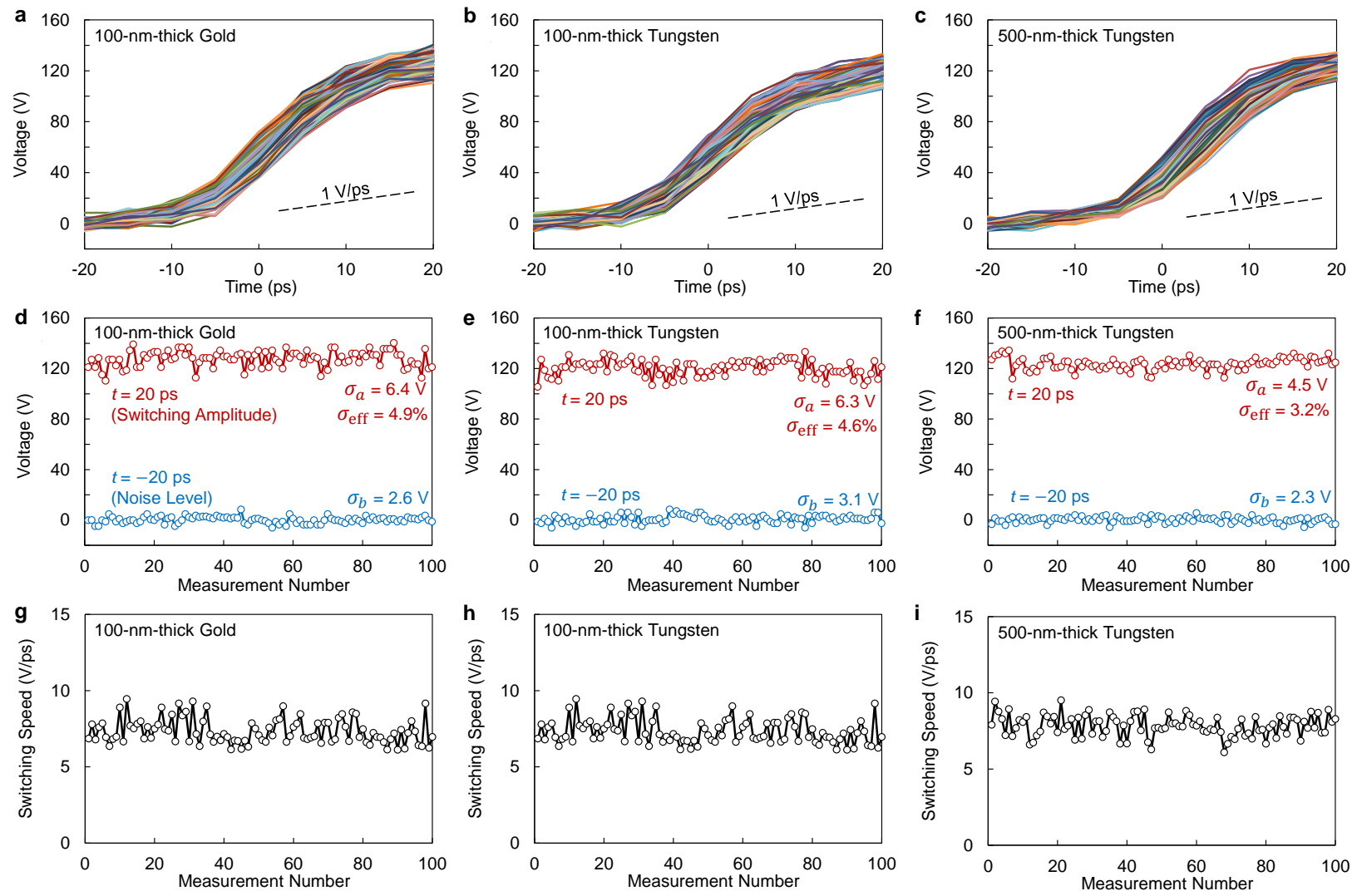

Extended Data Figure 4 | Statistics of the switching performance. 100 measured switching transients for 1000-nm-gap devices with a, 100-nm-thick gold, b, 100-nm-thick tungsten, and c, 500-nm-thick tungsten. d, e, and f, Measured switching voltage at $t=20 \mathrm{ps}$ (with standard deviation $\sigma_{a}$ ) and measured noise level at $t=-20 \mathrm{ps}$ (with standard deviation $\sigma_{b}$ ) corresponding to the waveforms shown in parts a, b, and c, respectively. The normalized effective standard deviation $\sigma_{\text {eff }}=\sqrt{\sigma_{a}^{2}-\sigma_{b}^{2}} / V_{\mathrm{sw}}$, where $V_{\mathrm{sw}} \sim 120 \mathrm{-V}$ is the switching voltage, are $4.9 \%, 4.6 \%$, and $3.2 \%$, for measured waveforms shown in parts $a, b$, and $c$, respectively. $\mathbf{g}, \mathbf{h}$, and $\mathbf{i}$, Measured maximum $d v / d$ corresponding to the measured waveforms shown in parts $a, b$, and $c$, respectively. Characterization of $\mathrm{d} v / \mathrm{d} t$ is more subjected to measurement errors because of the limited sampling time (5-ps/sample). It should be noted that the limited sampling time, generally leads to an underestimation of $d v / d t$, as the sampling does not necessarily pick the maximum of $d v / d t$. All the results presented are without de-embedding. 

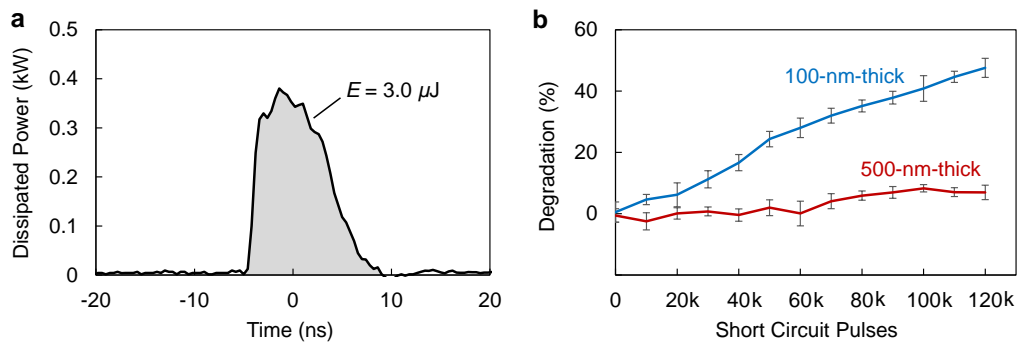

Extended Data Figure 5 | Lifetime evaluation under harsh switching condition. a. Dissipated power inside 700-nm-gap nanoplasma switch with tungsten pads under short circuit test resulting in the highest possible current density for lifetime measurements (high current density is the main driver for electromigration). Measurements showed energy and peak power dissipation of $3 \mu \mathrm{J}$ and $0.4 \mathrm{~kW}$ at each short circuit pulse. Such a high-power and energy dissipation are orders of magnitude higher than in practical applications. $\mathbf{b}$, Degradation with the definition of $\left(V_{\mathrm{TH}}[n]-V_{\mathrm{TH}}[0]\right) / V_{\mathrm{TH}}[0]$, where $V_{\mathrm{TH}}[n]$ is the threshold voltage at nth short circuit. The error bars show $\pm 2 \sigma$, where $\sigma$ is the standard deviation from 10 measurements. The obtained results for the proposed devices with sputtered tungsten pads show their capability of withstanding repetitive short circuits, without any specific optimization. The devices with a thicker pads (thus lower current density) provide a more stable performance even at very harsh condition, thus one could expect a very long lifetime in normal operations. In addition, electromigration has a solid background in silicon electronics with several demonstrated solutions, including the use of specific alloys, or single crystalline metals that result in nearly infinite lifetime even for sub-micrometer interconnections ${ }^{31}$. Thus even though the 100 -nm-thick devices showed a larger degradation in such extreme condition, they could also be useful in practical applications. 

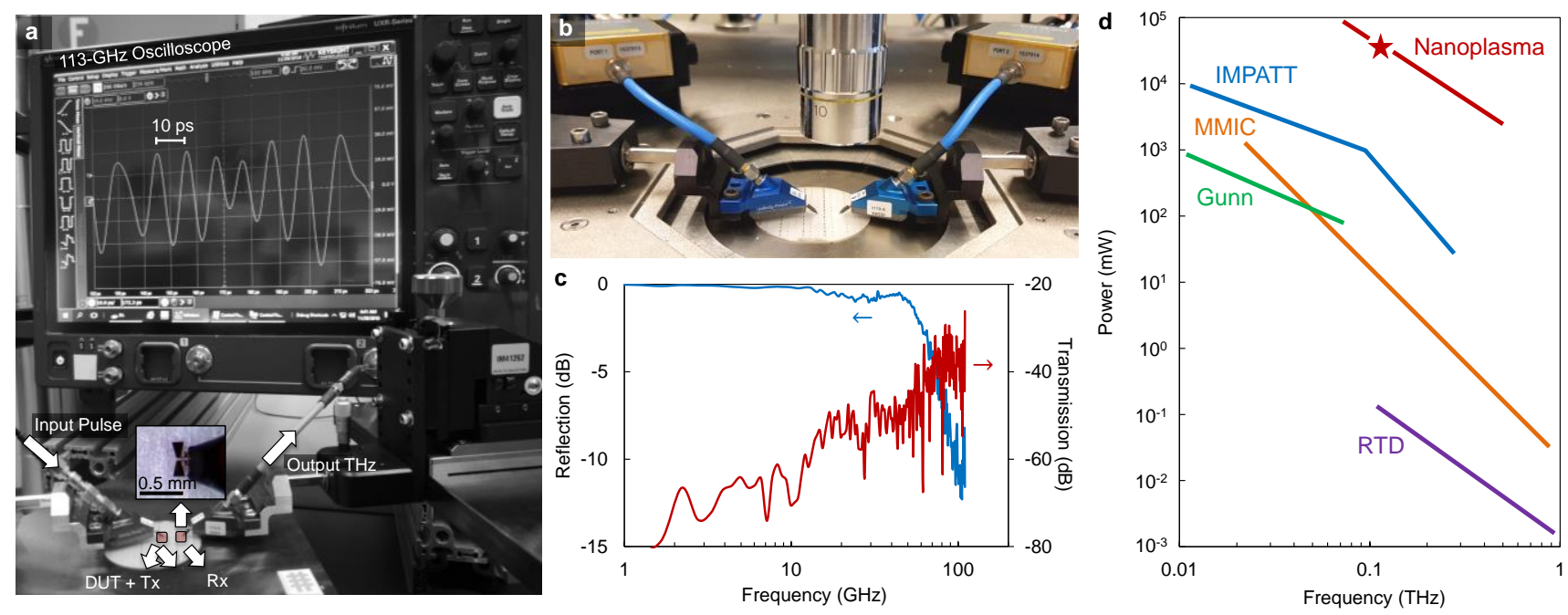

Extended Data Figure 6 | mm-wave/THz experimental set-ups and antenna characterization. a, Experimental set-up for characterizing mm-wave and $\mathrm{THz}$ radiation from the proposed devices integrated with bowtie antennas. A low speed input pulse charges the bowtie antenna, as a capacitance, until the voltage difference between two terminals reaches the threshold voltage. At this time, the nanoplasma switch turns ON in a very short time and excites the fundamental frequency of the bowtie antenna, as a resonator. The radiated wave is received by another bowtie antenna in front of the transmitter antenna. The receiver antenna is loaded by the $50-\Omega$ port of a UXR1102A Infiniium UXR-Series Keysight oscilloscope with 113-GHz bandwidth. b, Experimental set-up for characterizing 110-GHz antennas. c, Measured scattering parameters for the antennas. $\mathbf{d}$, Illustration of the obtained results in power/frequency sheet ${ }^{32,33}$, as well as the equivalent $P f^{2}=$ constant line. After de-embedding the effect of cables and RF probe, as well as $S_{21}$ of the antenna, we obtained average peak power of $50-\mathrm{W}$ at $109-\mathrm{GHz}$. 


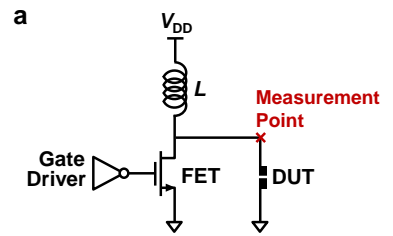

b

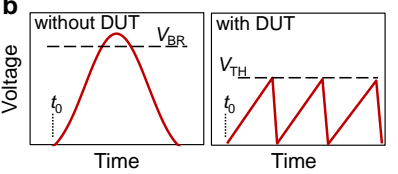

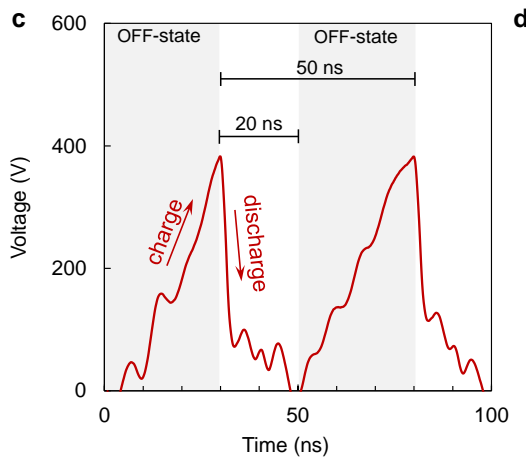

Extended Data Figure 7 | High repetition rate performance. a, Proposed circuit to demonstrate very high repetition-rate switching. The FET is ON for $t<t_{0}$, charging the inductor $L$. At $t=t_{0}$, the transistors turns OFF initiating a resonance between its output capacitance and inductor $L$. $\mathbf{b}$, Without connecting the DUT, a high-amplitude spike is generated. By connecting the DUT, when the voltage reaches the $V_{\text {TH }}$ of the switch, the DUT discharges the output capacitance. At this time, the inductor still has current, so it charges again the output capacitance. This charging/discharging process can continue up to several times, depending on the inductor current. c, Measured voltage waveform over DUT ( $g=6 \mu \mathrm{m})$ a small plasma recombination time $<20 \mathrm{~ns}$ to reconfigure the transistor back to its operation. This shows a high-switching frequency in the devices. The measured $<20$-ns recombination-time enables to achieve switching frequency up to $50-\mathrm{MHz}$ (depends on the duty cycle) at $390-\mathrm{V}$ (hollow red marker). In the current circuit however, limited the switching frequency to $20-\mathrm{MHz}$ (solid red marker). d, Benchmarking the obtained switching-frequency with state-of-the-art in solidstate electronics. These results shows the potential of the proposed devices, not only in ultra-fast $\mathrm{d} v / \mathrm{d} t$ transients, but also in switching frequency. For the references of the data points, please refer to the supplementary information. 


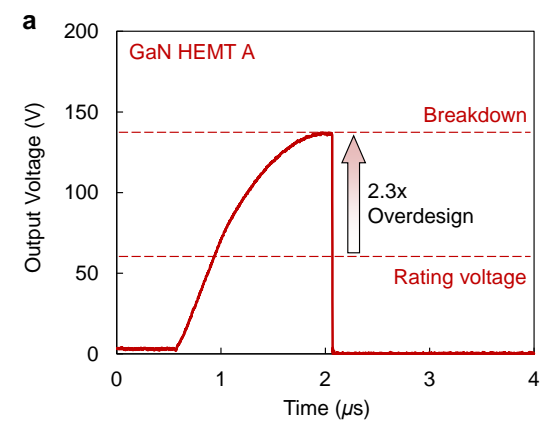

d

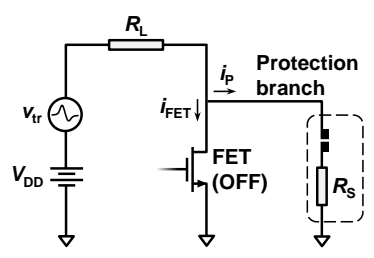

b
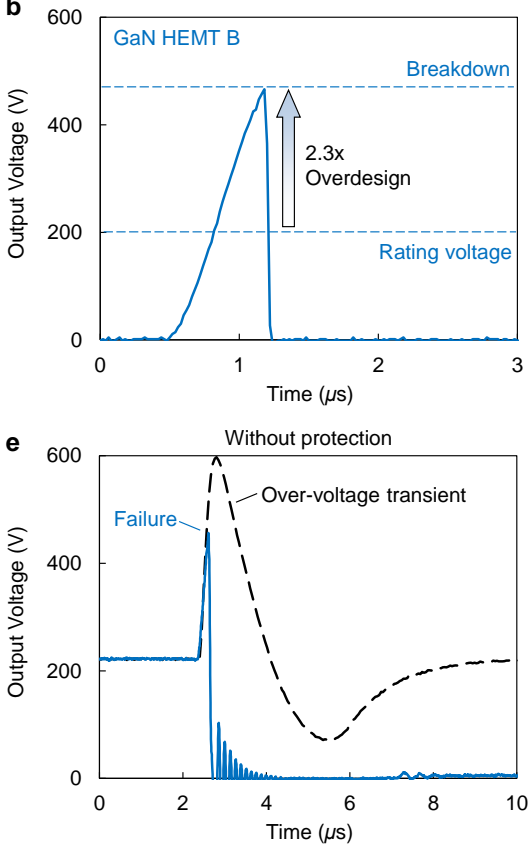

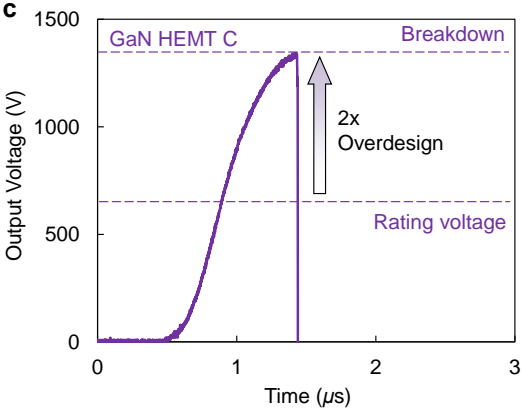

f

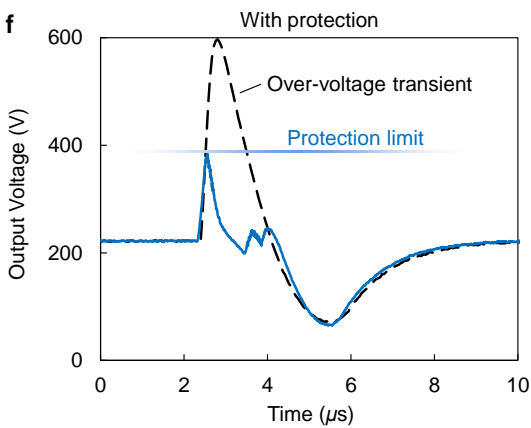

Extended Data Figure 8 | Application in over-voltage protection of devices and systems. RF ports of high-frequency systems need to have a unit to protect the system from over-voltage caused by electromagnetic interference (EMI), high-power RF radiations, etc ${ }^{34,35}$. The protection unit needs to be easily integrable, provide a fast action, a high-current capability, as well as a low parasitic capacitance. The proposed devices are well matched to this application, as they can discharge the over-voltage and retain their off-state in a short time. They can also be used to protect any electron device from over voltage causing hard breakdown and device failure. The voltage protection limit can be easily adjusted by the gap size. a, Breakdown test on a 60-V-rated GaN-on-Si HEMT (device A) resulting in a hard breakdown voltage ( $V_{B R}$ ) of 140-V. b, Breakdown test on a $200-V$-rated GaN HEMT (device B) resulting in $V_{B R}=470 \mathrm{~V}$. c, Breakdown test on a 650-V-rated GaN HEMT (device C) resulting in $V_{B R}=1340$ V. Since there is no avalanche characteristics in GaN HEMTs ${ }^{36}$, the manufacturers have to over design the devices to ensure a safe operation. This leads to a much higher ONresistance which considerably increases the amount of losses in power converters. By integrating the proposed devices inside the package, it is possible to protect the device from over-voltages to eliminate the over design which eventually leads to lower ON-resistance. d, Proposed circuit to demonstrate the protection application for a FET from a hard-breakdown. The FET is in the OFF-state holding voltage $V_{D D}$, and an over-voltage $V_{\text {tr }}$ is applied. When the voltage over device is lower than the protection limit $\left(V_{T H}\right)$, the plasma device is OFF, resulting in ultra-low parasitics (Extended Data Fig. 3). As a result, the protection branch current (iP) is completely negligible with respect to the FET current (iFET); therefore, the protection branch do not affect the normal operation of the device. However, when the drain-source voltage becomes larger than $V_{\mathrm{TH}}$, the plasma device discharges the extra energy, protecting the device from hard breakdown. The series resistance $R \mathrm{~s}=1 \mathrm{k} \Omega$ is used for smoothing the discharge process. The load resistance $(R \mathrm{~L})$ was considered to be $1-\mathrm{k} \Omega$. To demonstrate the functionality of this method, we used GaN HEMT B biased at $V_{\mathrm{DD}}=220 \mathrm{~V}$ and plasma device with $V_{\mathrm{TH}}=$ $380 \mathrm{~V}$. e, Output voltage without protection branch, showing a hard breakdown at $460 \mathrm{~V}$. f, Output voltage with protection branch. The plasma device discharges the over-voltage, limiting the output voltage to $385 \mathrm{~V}$. The protection limit $\left(V_{\mathrm{TH}}\right)$ can be easily adjust by tuning the gap distance. 